\title{
Aminopyrene functionalized reduced graphene oxide as a supercapacitor electrode
}

\begin{abstract}
In this work, we report on the structural and electrochemical properties of aminopyrene functionalized reduced graphene oxide (Ap-rGO) for its suitability as a supercapacitor electrode. The Ap-rGO is prepared by sonicating a suspension of $\mathrm{rGO}$ with aminopyrene and the filtered sediment is subjected to spectroscopy studies and electrochemical studies. Spectroscopy studies reveal the successful functionalization of aminopyrene onto Ap-rGO through $\pi-\pi$ interactions. Electrochemical analyses of Ap-rGO show a substantial increase in the specific capacitance for Ap-rGO (160 F g-1 at $5 \mathrm{mV} \mathrm{s}-1)$ compared to the nonfunctionalized $\mathrm{rGO}(118 \mathrm{~F} \mathrm{~g}-1$ at $5 \mathrm{mV} \mathrm{s}-1)$. The enhancement is shown to be the pseudocapacitance arising from the electron donating effect of the amine group and the electron accepting effect of rGO, which enable facile electron transfer between the surfacebound amine group and rGO. The Ap-rGO has desirable charge storage properties such as low series resistance $(0.4 \Omega)$ and superior cycling stability ( $85 \%$ after 5000 cycles). Furthermore, the Ap-rGO has 1.5 fold higher energy density than the non-functionalized rGO electrode, thereby making it suitable as a deployable supercapacitor electrode.
\end{abstract}

